\title{
AID for Blind Person
}

\author{
Jitendra Kumar Shukla, \\ Final Year Student, M.Tech, Electronics Design and Technology, \\ National Institute of Electronics \& Information Technology, Aurangabad, Maharashtra, India, \\ Warsha Kandlikar \\ Scientist ' $C$ ', Department of Electronics and IT \\ National Institute of Electronics \& Information Technology, Aurangabad, Maharashtra, India,
}

\begin{abstract}
For blind people navigation is the hard task in their day to day life, So we have developed navigate assistance for blind person a compact system with a hardware raspberry pi, camera and ultrasonic sensor is formed. The proposed system is mounted on waist. This system gathers information for blind person to navigate. In this system if there is an obstacle in the path the beep sound will be played, afterward a camera will start and using image processing system will detect whether the obstacle is moving or non moving and it coming toward are going away by comparing current frame with reference frame if object coming toward then intensity of frame continuously increase. Moving object detection is based on Gaussian mixture model and Computer vision tool box. Moving object detection involves identification of an objecting consecutive frames and object detection is used to monitor the movements with respect to the region of interest. This system is implemented in two parts, one is using background and foreground detection in MATLAB and second part is Raspberry pi python programming.
\end{abstract}

Keywords - Moving object detection, tracking, Gaussian mixture model estimation, morphological operation, blob analysis, Raspberry pi. Ultrasonic sensor background and foreground detection

\section{INTRODUCTION}

The main objective of this project is to navigate the blind person. Navigation means when blind person is moving on street this project tells whether the object is coming towards him or that object is stationary in front of him. This project consists of software simulation on MATLAB and Python and can be implemented as hardware on raspberry pi board. Moving object analysis and estimation is done by Gaussian mixture model in MATLAB, First we take average of many no of video frame

- $B(x$ y $t)=1 / N($ sum of $V(x$ y $t-i))$

- Where $\mathrm{V}(\mathrm{x}$ y $\mathrm{t}-\mathrm{i})$ is a video frame for $\mathrm{i}=0,1,2,3-------$

After that we take background and foreground detection

A Gaussian Mixture Model (GMM) is a parametric probability density function represented as a weighted sum of Gaussian component densities. GMMs are commonly used as a parametric model of the probability distribution of continuous measurements or features in a biometric system, such as vocal-tract related spectral features in a speaker recognition system. GMM parameters are estimated from training data using the iterative Expectation-Maximization (EM) algorithm or Maximum A Posterior (MAP) estimation from a well-trained prior model.

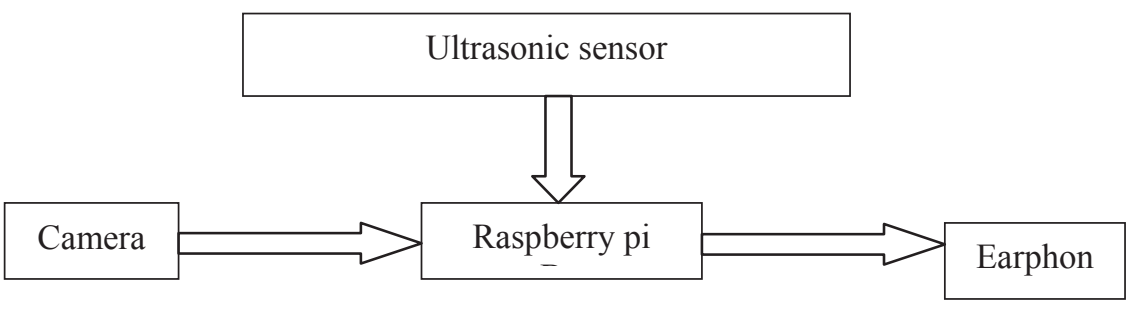

Fig.1. Block diagram of AID for Blind People 
TABLE I COMPARISON OF ULTRASONIC SENSOR

\begin{tabular}{|c|c|c|c|}
\hline Parameters & $\begin{array}{c}\text { Ping)) }) \\
\text { Ultrasonic Distance Sensor }\end{array}$ & $\begin{array}{c}\text { Robokits RKI-1540 Sensor } \\
4 \mathrm{M}\end{array}$ & TI Ultrasonic Transducer pair 40KHz \\
\hline $\begin{array}{c}\text { Center } \\
\text { frequency }\end{array}$ & $40 \mathrm{KHz}$ & $40 \mathrm{KHz}$ & $40 \mathrm{KHz}$ \\
\hline Range Approx & $2 \mathrm{~cm}$ to $3 \mathrm{~m}$ & $2 \mathrm{~cm}$ to $4 \mathrm{~m}$ & Up to $18 \mathrm{~m}$ \\
\hline $\begin{array}{c}\text { Power } \\
\text { requirement }\end{array}$ & $+5 \mathrm{VDC} 35 \mathrm{~mA}$ & $+5 \mathrm{VDC} 15 \mathrm{Ma}$ & $+5 \mathrm{~V} 15 \mathrm{~mA}$ \\
\hline Beam Angle & 15 degree max & Max 15 degree & Max 55 degree \\
\hline
\end{tabular}

TABLE II RANGE OF PING ULTRASONIC SENSOR FOR DIFFERENT MATERIAL

\begin{tabular}{|c|c|c|c|}
\hline $\begin{array}{c}\text { Obstacle } \\
\text { surface }\end{array}$ & \multicolumn{3}{|c|}{ Detection range in cm } \\
\hline & Test1 & Test2 & Test 3 \\
\hline Metal & 290 & 275 & 285 \\
\hline Wood & 200 & 202 & 206 \\
\hline Human body & 180 & 189 & 185 \\
\hline
\end{tabular}

Ultrasonic sensor transmits waves when the pulse of 10 microsec is given to the trigger input of ultrasonic. It has two transducer one for transmitting and second for receiving the reflected waves, after triggering it will transmit a eight $40 \mathrm{KHz}$ waves if there is some object then the waves reflected back, and receiver pin get high of $5 \mathrm{~V}$ but raspberry pi accept only $3.3 \mathrm{~V}$ so use voltage divider after that video processing start, Filtering process removes the noise and finally blob analysis is employed to identify object. We only display boundary box that is greater than a certain size and the size is determined according to the object to be detect. After completion of MATLAB work we convert the MATLAB coding into the raspberry pi board language.

First ultrasonic sensor start transmitting continuous signal when it detect object then once beep sound will play and camera start in video mode and start capturing video if frame intensity remain stationary than object is stationary and if frame intensity change than object is moving and prerecorded sound will play. If frame size increase than object coming toward other vise going away

\section{ALGORITHM}




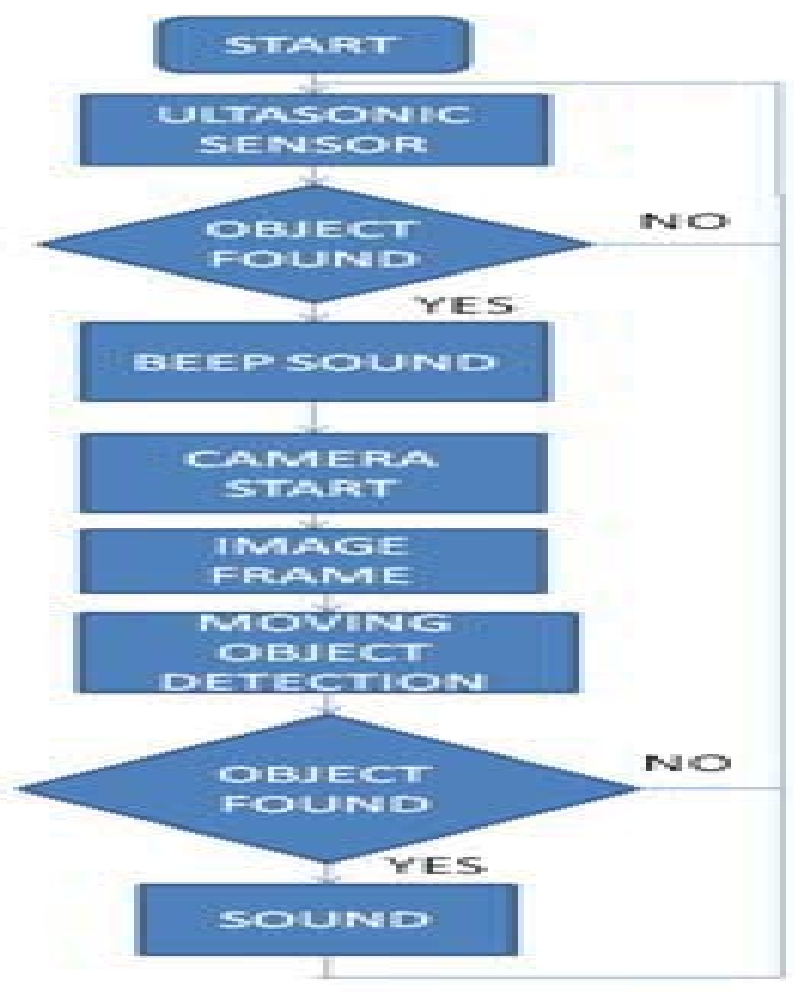

Fig.2. Flow Diagram of Proposed Algorithm

In the developed algorithm we are going step-by-step to get the expected result a video input is selected from static camera, further on that video some pre-processing operation are done i.e. convert the color video frame into grayscale video frame. Due to cameras auto white balance and the effect of sudden environment intensity changes, mean of every frame is calculated on gray scale format. After that we take average of $\mathrm{N}$ number of frames than take it as reference and compare with current frame and set background and find foreground, From these we can calculate the velocity of motion vectors, out of all the pixel of frame only moving pixels are moving object. During filtering operation some holes are created in frames to fill these holes and prevent the detection mistakes morphological operation i.e. closing and erode are implemented. Now motion object are detected but many of them are not interested, blob analysis help us to cluster objects and filter out objects which are be based on blob size. Drawing bounding boxes around the object is last segment of the algorithm.

\section{GAUSIAN MIXTURE MODEL}

First each picture is characterized by its intensity in the RGB color space. Then, the probability of observing the current pixel value is considered given by the following formula in the multidimensional case:

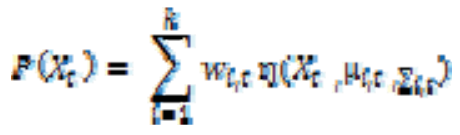

Where the parameters are $\mathrm{K}$ is the number of distributions, $w$ is a weight associated to the $\mathrm{i}^{\text {th }}$ Gaussian at time $\mathrm{t}$ with mean $m_{i, t}$ and standard deviation has a Gaussian probability density function: 


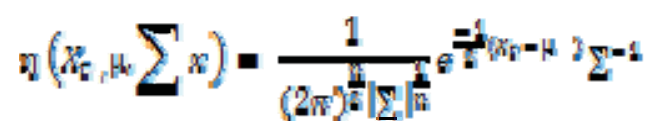

For computational reasons, Stauffer and Grimson [1] assumed that the RGB color components are independent and have the same variances. So, the covariance matrix is of the form:

$$
\Sigma_{n, t}=s^{2}{ }_{h t} \mathrm{I}
$$

So, each pixel is characterized by a mixture of K Gaussians. Once the background model is defined, the different parameters of the mixture of Gaussians must be initialized. The parameters of the MOG's model are the number of Gaussians K, the weight associated to the ith Gaussian at time t, the mean and the covariance matrix.

\section{IMAGE SEGMENTATION}

Thresholding is the simplest method of image segmentation, from gray scale image thresholding can be used to create binary images, So that the objects of interest can be highlighted by fixing a threshold limit. In tracking algorithm the content of each frame is read and the background is estimated. During thresholding process individual pixels in an image are marked as 'object' pixels, if their value is greater than some threshold value i.e. background pixel. An object pixel is given a value of 1 while a background pixel is given a value of 0 .finally binary image created by coloring each pixel white or black. ${ }^{[7]}$

The key parameter in the thresholding process is the choice of the threshold value. We can manually choose threshold value or threshold algorithm can compute a value automatically, simple method would be to choose the mean or median value.IN noiseless image with uniform background and object values, the mean or median will work well as the threshold. A more sophisticated approach might be to create a histogram of the image pixel intensities and use the valley point as the threshold. The histogram approach assumes that there is some average value for the background and object pixels, but that the actual pixel values have some variation around these average values. However, this may be computationally expensive, and image histograms may not have clearly defined valley points, often making the selection of an accurate threshold difficult. ${ }^{[1][7]}$

\section{NOISE FILTERING}

Because the camera is not stationary, every time the person has to stop if the object detected by ultrasonic. But since the person can stand like statue, there is some movement, and this cause an error in object detection. So we decided to reduce this error by filtering in MATLAB we have morphological opening to remove noise in the foreground.

Morphological operations are performed to extract significant features from images that are useful in the Representation and description of the shapes in the region; mostly used in image segmentation and pattern recognition. In the proposed system we used morphological operation to remove the unwanted pixel value that is called noise because small pixel value can from a object so the appearance of object is not destroyed. The following is the definition of morphological closing operation and the applied structural element B.

$$
\mathrm{P} \bullet \mathrm{B}=(\mathrm{P} \oplus \mathrm{B}) \oplus \mathrm{B}
$$

Where, 


$$
B=\left|\begin{array}{lll}
0 & 0 & 1 \\
0 & 1 & 0 \\
1 & 0 & 0
\end{array}\right|
$$

The matrix $P$ which includes moving vehicle information is obtained through threshold segmentation. [4][6]

\section{BLOB ANALYSIS}

In the area of computer vision blob detection refers to visual modules that are aimed at detecting points and/or regions in the images that differ in properties like brightness or color compared to surrounding.

Blob analysis provides complementary information about region which is not obtaining from edge detectors or corners detectors. Blob detection used to obtain region of interest. Blob analysis can be used to detect any kind of 2- dimensional shapes of an image. The detection is based on spatial characteristics using certain criteria. In many applications where the computation is time consuming, one cause blob analysis to eliminate blobs that are of no interest based on certain spatial characteristics and keep only the relevant blobs for further analysis. The blobs which satisfied our system are vehicles. Other useless blobs are removed by setting limitations on the relative features in the algorithm.

Here we used blob analysis for finding 'Bounding Box Output Port Area Output Port Centroid Output Port Minimum Blob Area', but we used only Minimum blob area to calculate the object. If the area value is greater than set value, then object is detected otherwise it is consider as zero.

\section{EXPERIMENTAL RESULT}

In this section we show the experimental results using the stationary camera and moving object. Before applying of Gaussian mixture estimation on frames, the image format is converted from RGB to gray because Intensity measurements act well on gray-scale frames. Depends on tracking flow steps, the proper optical flow estimation has been applied. Then, the Median filter is performed to reduce noise corruptions.

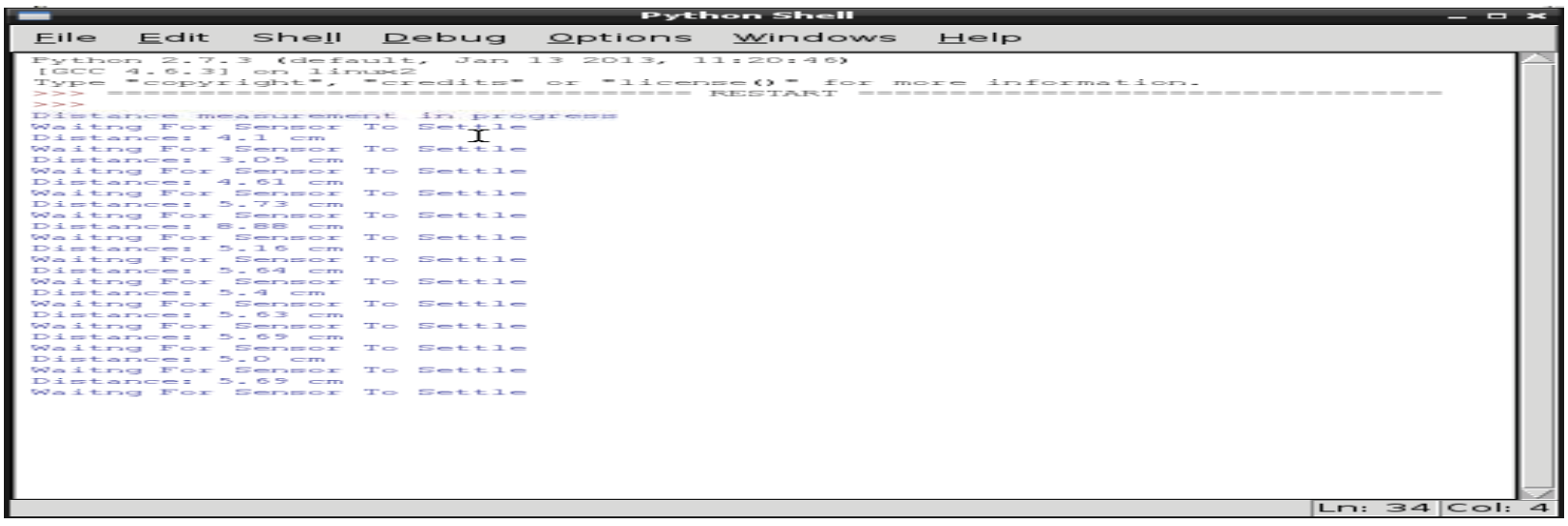

Fig.3. Ultrasonic result 


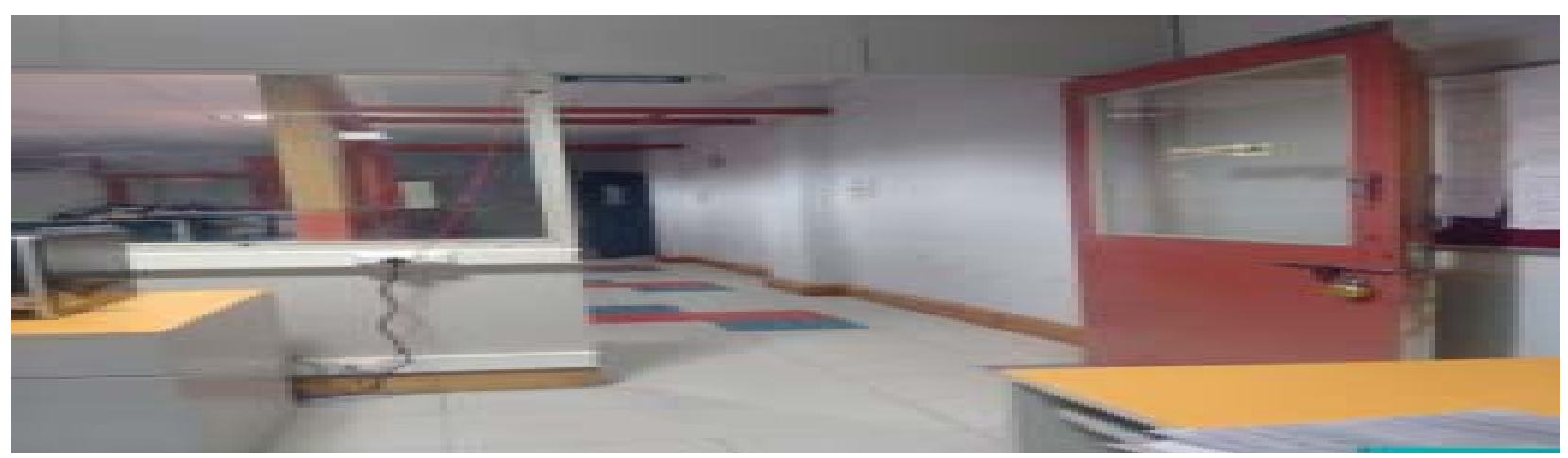

Fig.4. Reference Frame

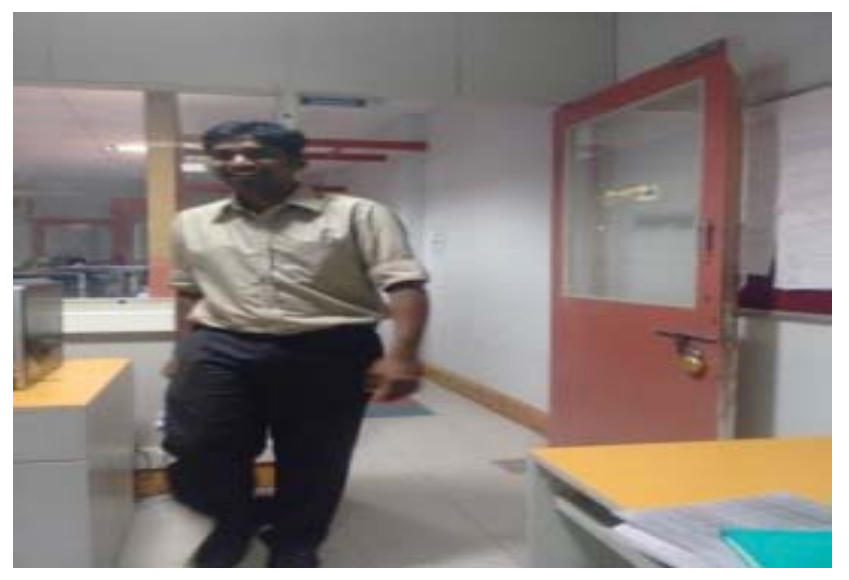

Fig.5. Current Frame

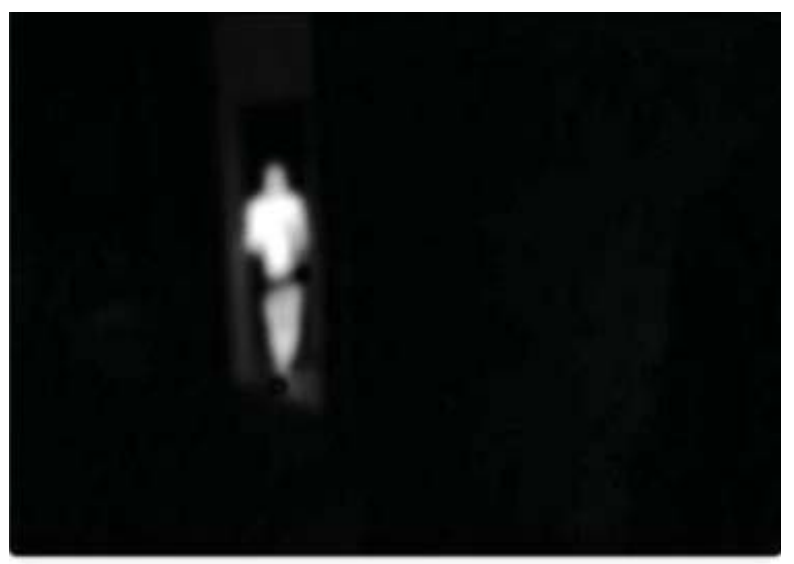

Fig.6. Background Detection

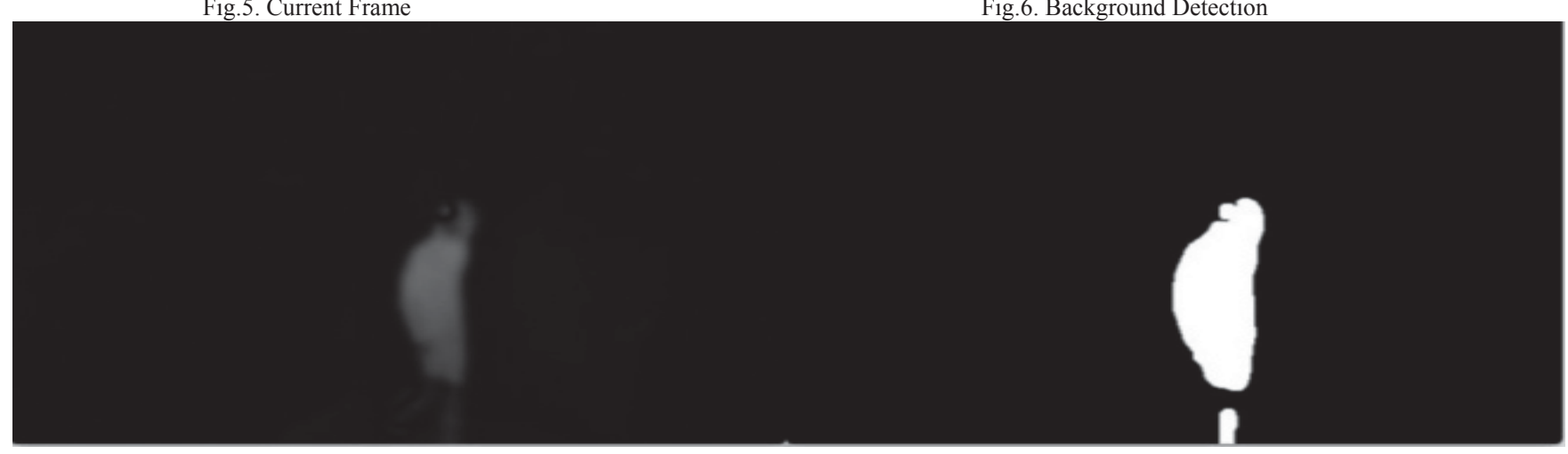

Fig.7. Background and Foreground Detection

\section{CONCLUSION}

We have tracked the moving object in video using background and foreground algorithm, this system employs various methods to detect, filtering, and segmentation and tracking objects. We used most suitable method of Gaussian mixture model estimation, to detect the moving object and object coming toward and going away by intensity changes of frame. The morphological close extracted significant features of region shapes from binary images and then blob analysis introduced. The great advantage of blob analysis is the low computation cost, the system removes unwanted motion object which are not vehicles in the images. After detecting the object our 
MATLAB work is completed, we now convert the MATLAB code into raspberry board language which is nothing but in the python language.

\section{ACKNOWLEDGMENT}

I take this opportunity to express profound gratitude and deep regards to my project guide Mrs. Warsha Kandlikar for his exemplary guidance, monitoring and constant encouragement throughout. The help and guidance given by him time to time is a valuable asset to my paper.

I also take this opportunity to express a deep sense of gratitude to Mr. John G. and all the staff members for their cordial support and valuable information, which helped me in completing this task through various stages.

Lastly, we thank almighty, our parents, friends for their constant encouragement without which this paper would not be possible.

\section{REFERENCES}

[1] LeFloch, D. Real-time people counting system using video camera.Master's thesis, Gjoevik University College, University de Bourgogne,2007.

[2] Object Tracking: A Survey, AlperYilmaz, Omar Javed, Mubarak Shah

[3] Brown, L. M. View independent vehicle/person classification. Technical report, Proceedings of the ACM 2nd international workshop on Video surveillance \& sensor networks, pages 114-123, 2004.

[4] Masamitsu Tsuchiya, H. F. Evaluating feature importance of object classification in visual surveillance. Technical report, The $18^{\text {th }}$ International Conference on Pattern Recognition (ICPR'06), Vol 2, pages978-981, 2006.

[5] Ying-Li Tian, M. L. \& Hampapur, A. Robust and efficient foreground analysis for real-time video surveillance. Technical report, Proceedings of the 2005 IEEE Computer Society Conference on Computer Vision and Pattern Recognition (CVPR'05), Vol 1, pages 11821187, 2004.

[6] Omar Javed, M. S. Tracking and object classification for automated surveillance. Technical report, Proceedings of the 7th European Conference on Computer Vision- Part IV, pages 343-357, 2002.

[7] Silar, Z., and M. Dobrovolny. "Comparison of two optical flow estimation methods using Matlab."Applied Electronics (AE), 2011International Conference on. IEEE, 2011

[8] Brijesh Patel and Neelam Patel ' motion detection based on multi frame video under surveillance system'

[9] David Moore 'a real world system for human motion detection and tracking

[10] G.johansson, 'Visual perception of biological motion and a model for its analysis 